\title{
High-Altitude Astrophotography and Investigating the Ionosphere 2020 WSGC ELIJAH High-Altitude Balloon Payload
}

\author{
Narisse King, Rebecca Joyce Alibio \\ Milwaukee School of Engineering
}

\begin{abstract}
The objective of the 2020 WSGC Elijah High-Altitude Balloon Payload Team was to design a payload intended to collect data during the total solar eclipse taking place in April 2024. The COVID-19 pandemic posed unique challenges to the research team, primarily restricting their correspondence to remote meetings and preventing them from fabricating a payload to launch for flight testing. Ground-based experimentation was conducted to analyze the viability of each of the payload modules to be constructed and launched in a future research term. Two of the four designed experiments are elaborated on in this document: an astrophotography and luminance tracking system to image celestial objects and determine the visibility of deep-space objects, and a surveillance system to identify and measure variation in magnetic fields and solar radiation in the ionosphere.
\end{abstract}

\section{Introduction}

The Elijah High-Altitude Balloon Research Student Satellite Initiative is an undergraduate research program established by the Wisconsin Space Grant Consortium (WSGC) ${ }^{1}$ to provide students in the STEM field the opportunity to gain research experience via developing a payload for a high-altitude balloon. The COVID-19 pandemic prevented the team from physically constructing a payload for flight, so research was limited to ground-based testing as a basis for future work. Two subsections of the experimental process, astrophotography and ionospheric surveillance, will be elaborated upon to test scientific theory and confirm viability of the experiments for flight. The remaining two subsections, analyzing the speed of sound as it relates to atmospheric variables and the detection of cosmic rays (muons) during flight, were also tested in depth but are omitted for brevity.

\section{Astrophotography}

The astrophotography sub-team's goals were to develop a way to remotely image the sun and surrounding space during the total eclipse as well as find mathematical relationships between the luminance of the sun and the visibility of deep-space objects.

Introduction. A total eclipse provides a unique opportunity to capture features of space that are difficult or impossible to capture in ordinary circumstances, such as the sun's coronasphere. As astrophotography is the most accessible method of recording space, this led the team to conceptualize a remote photography system that would take advantage of both the total eclipse phenomenon and the unique spatial perspective of a high-altitude balloon. Since the instruments of the payload will rise above cloud cover and light pollution, there is a rare opportunity to see space with an unobstructed view. As sky luminance changes from daylight to totality, an additional opportunity presents itself to see objects in space that are invisible during

\footnotetext{
${ }^{1}$ The authors would like to thank the WSGC for providing this opportunity and the requisite funding, as well as the Milwaukee School of Engineering for providing their 3D-printing facilities.
} 
usual daylight hours. This sub-team sought to find out whether or not deep-space objects such as clusters and galaxies are visible in totality based on the luminance of the sky as measured in lux.

Methods. The remote photography system included three distinct image modes: infrared light, visible light (420-680nm), and solar imagery. This was achieved using a Canon PowerShot ELPH135 modified to capture infrared light and two external filters, one to block all light except the visible spectrum and a solar filter to photograph the sun while preventing damage to the camera. It is assumed that the infrared band imaged during testing is approximately $725-1000 \mathrm{~nm}$, given that camera sensors are typically sensitive only up to $1000 \mathrm{~nm}$ and the "hot mirror" used to internally filter infrared light (removed during the conversion process) filters light below roughly $725 \mathrm{~nm}$. To incorporate the external filters, a custom mount was modeled in OnShape, then 3D-printed for use. The final version of the model is shown in Figure 1.

To rotate the filter housings and utilize all three modes of photography, code was written in the Arduino IDE to activate servos attached to the mount; once a picture was taken, one or both servos would rotate the corresponding filter in or out of the lens's field of view to switch to the next imagery mode.

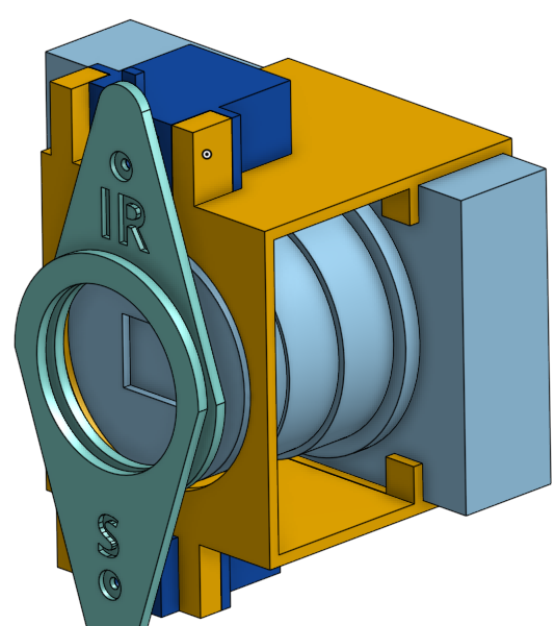
Additionally, the Canon Hacker Development Kit (CHDK) was utilized in order to trigger the camera to shoot without manual input.

Sky luminance testing was conducted utilizing a light sensor system created with a photoresistor controlled by the Arduino IDE. This code recorded the photoresistor readings and converted the values to lux units based on voltage-to-lux values obtained using a CdS photocell with a $3.3 \mathrm{~V}$ input scale, converted to a $5 \mathrm{~V}$ input scale with the equation:

$$
V_{f}=\left(V_{i} / 3.3\right) \times 5
$$

Figure 1: OnShape model of the final camera mount.

Includes the mount (gold), camera (light blue),

servos (dark blue), and filter housings (teal).

This created the voltage-to-lux conversion chart as shown below:

Table 1: Voltage-to-Lux conversion chart, 0-3.3V scale (left) and 0-5V scale (right) Note: Data are from Hamblen (2015)

\begin{tabular}{|r|r|r|r|r|}
\hline \multicolumn{1}{|r|}{ Volt } & Lux & \multicolumn{1}{|c|}{ Volt } & \multicolumn{1}{|c|}{ Lux } \\
\hline $0.412^{\mathrm{a}}$ & $11^{\mathrm{a}}$ & 0.6242424242 & 1 \\
\hline $1.34^{\mathrm{a}}$ & $10^{\mathrm{a}}$ & 2.03030303 & 100 \\
\hline $1.65^{\mathrm{a}}$ & $100^{\mathrm{a}}$ & 2.5 & 1000 \\
\hline $3.2^{\mathrm{a}}$ & $1000^{\mathrm{a}}$ & 4.848484848 & \\
\hline
\end{tabular}




\begin{tabular}{|c|c|c|c|}
\hline $3.26^{\mathrm{a}}$ & \begin{tabular}{l|l}
$10000^{\mathrm{a}}$ \\
\end{tabular} & 4.939393939 & 10000 \\
\hline
\end{tabular}

The luminance experiment ran in two one-hour blocks, one during peak daylight hours within the zone of 10:00AM CDT to 2:00PM CDT, and one during the thirty minutes before and after sunset. Data collected from this program was compiled into Google Sheets, then graphed for extrapolation.

Results. Numerous benchmark photos were taken in all imagery modes to calibrate the camera settings that would likely be effective during a launch. Below are three images taken in the visible light spectrum varying from bright to low daylight taken on the same day (July 28):

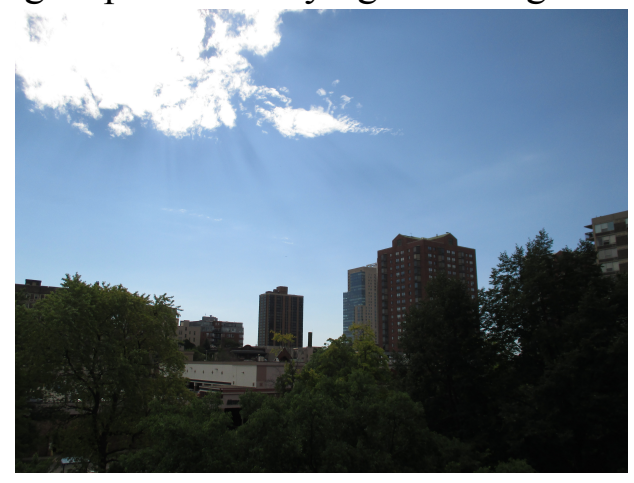

Fig. 2: Benchmark 1, Bright daylight, mostly clear sky

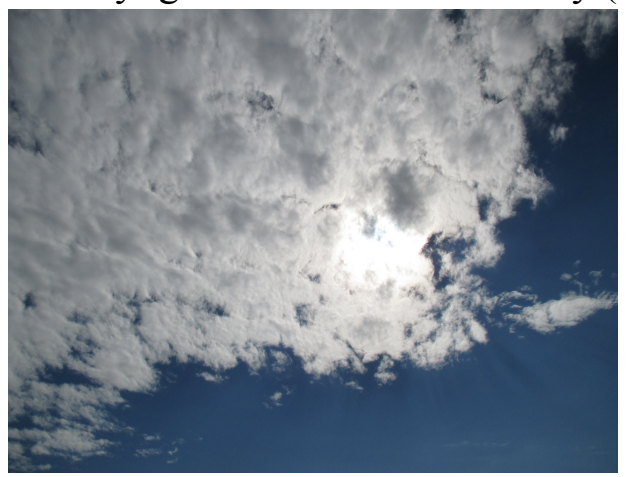

Fig. 3: Benchmark 2, Partially cloudy

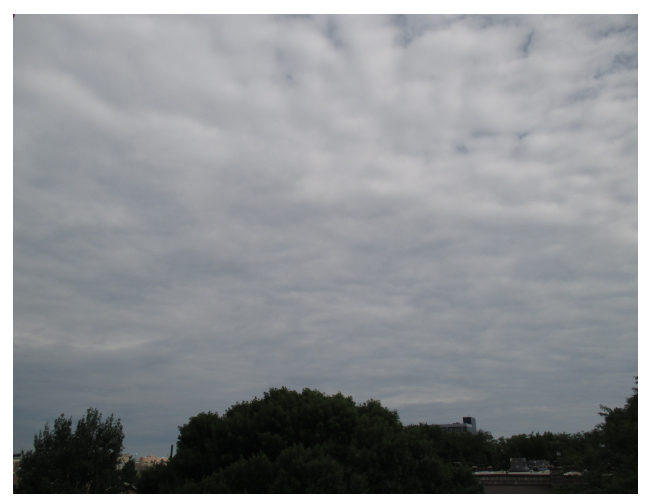

Fig. 4: Benchmark 3, Cloudy

Table 2: Camera settings for daylight imagery

\begin{tabular}{|l|c|c|c|c|}
\hline & ISO & $\begin{array}{c}\text { F-stop } \\
\text { (Aperture) }\end{array}$ & $\begin{array}{c}\text { Shutter Speed } \\
(\mathrm{s})\end{array}$ & $\begin{array}{c}\text { Focal Length } \\
(\mathrm{mm})\end{array}$ \\
\hline Benchmark 1 & 100 & 9 & $1 / 320$ & 5 \\
\hline Benchmark 2 & 100 & 9 & $1 / 1250$ & 5 \\
\hline Benchmark 3 & 100 & 9 & $1 / 400$ & 5 \\
\hline
\end{tabular}


Comparison images of celestial objects, namely the sun and Mars, were taken in the infrared and visible light modes to compare visibility (infrared light sensed by the camera registered as magenta/pink visible light):

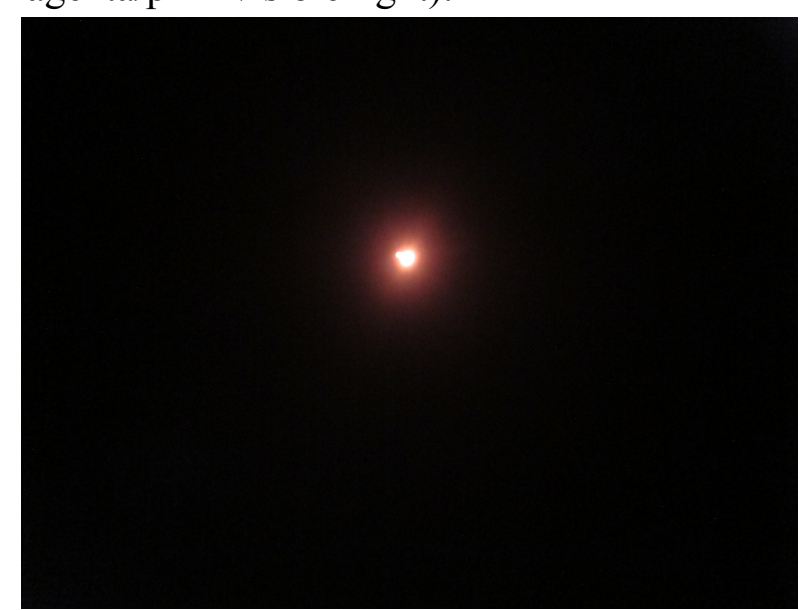

Fig. 5: Benchmark 4: Sun visible light

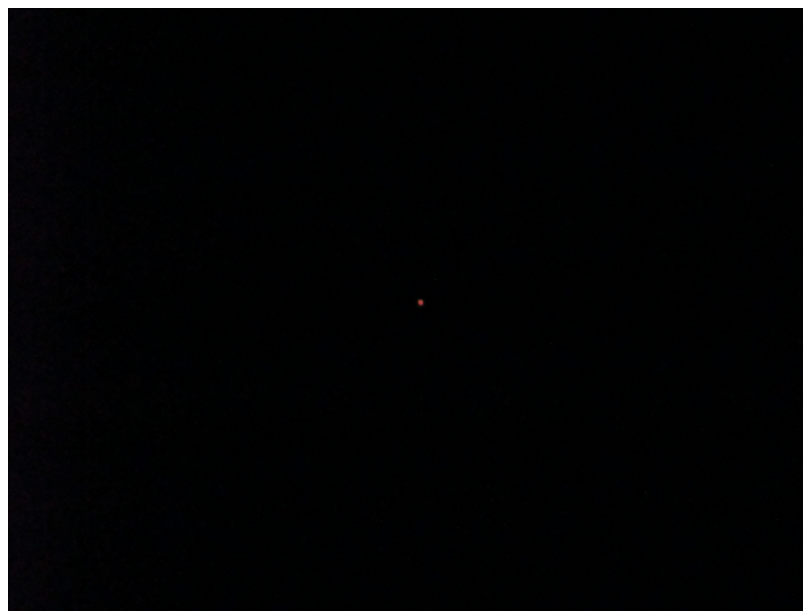

Fig. 7: Benchmark 6, Mars, visible light

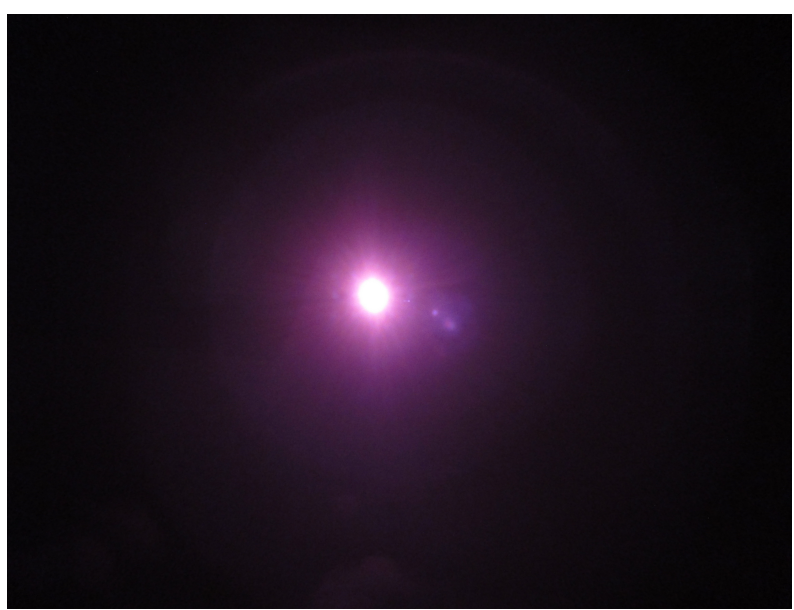

Fig. 6: Benchmark 5, Sun, infrared

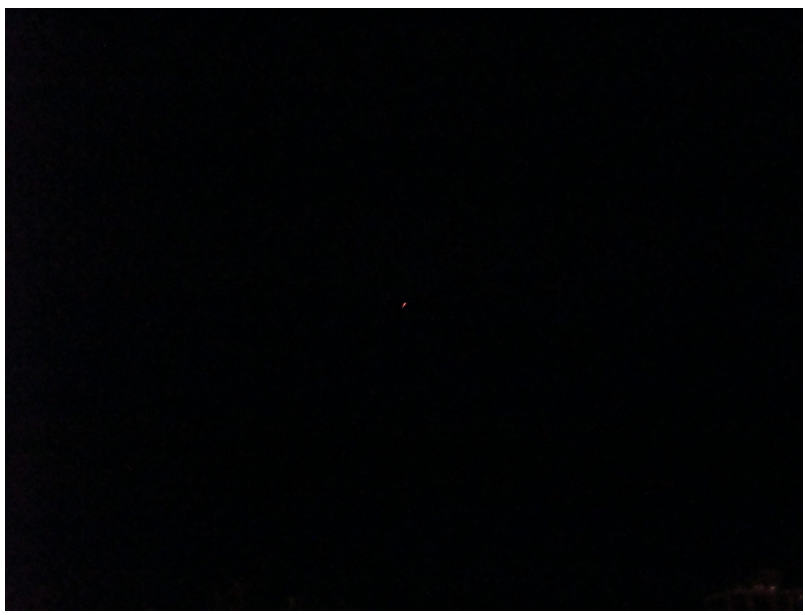

Fig. 8: Benchmark 7, Mars, infrared Infrared light emitted by the sun varies from $50 \%$ of total light output at the top of the atmosphere, to $53 \%$ at ground level; visible light is less significant at a variance of $40 \%$ to $44 \%$ (University of Tennessee Institute of Agriculture, n.d.). As shown in the benchmark pictures, the radius of infrared light is noticeably wider than the radius of visible light. It follows that the infrared image of Mars would leave the planet near-invisible as reflected light from the sun largely constitutes visible light, showing the planet in its usual red hue.

For the luminance experiment, data was collected in Waukegan, IL during the hours of 10:15AM to 11:15AM CDT on August 7, and 7:29PM to 8:29PM CDT on August 6. The light sensor took a sample every thirty seconds during these timeframes. Averaging the samples collected during peak daylight with mostly clear skies provided a daytime luminance of 12006.77 lux. As the sky brightness of the path of totality is estimated to be roughly 1/100,000th of daylight brightness, or twilight conditions, the daytime luminance figure was multiplied by that factor to find a projected totality luminance of 0.12 lux (Pettengill, 2017). 
During sunset, luminance decreased steadily, graphed below. The line of best fit is represented as a power-four polynomial:

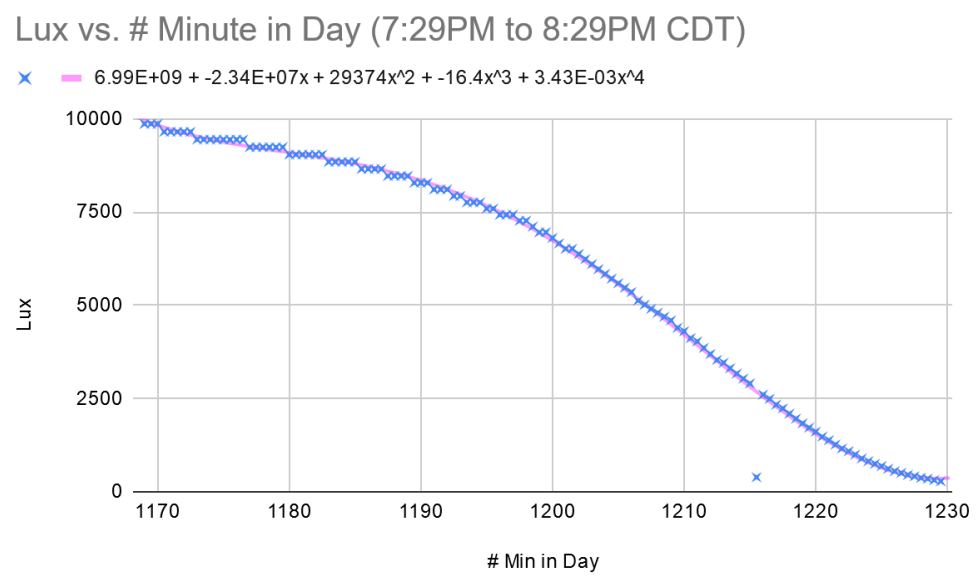

Fig. 9: Lux vs. Time (as converted to the number of the minute in the day) during sunset

As luminance dropped below 1000 lux, the slope of the graph changed greatly, shown separately:

Lux vs. \# Min. in Day (Night, < 1000 Lux)

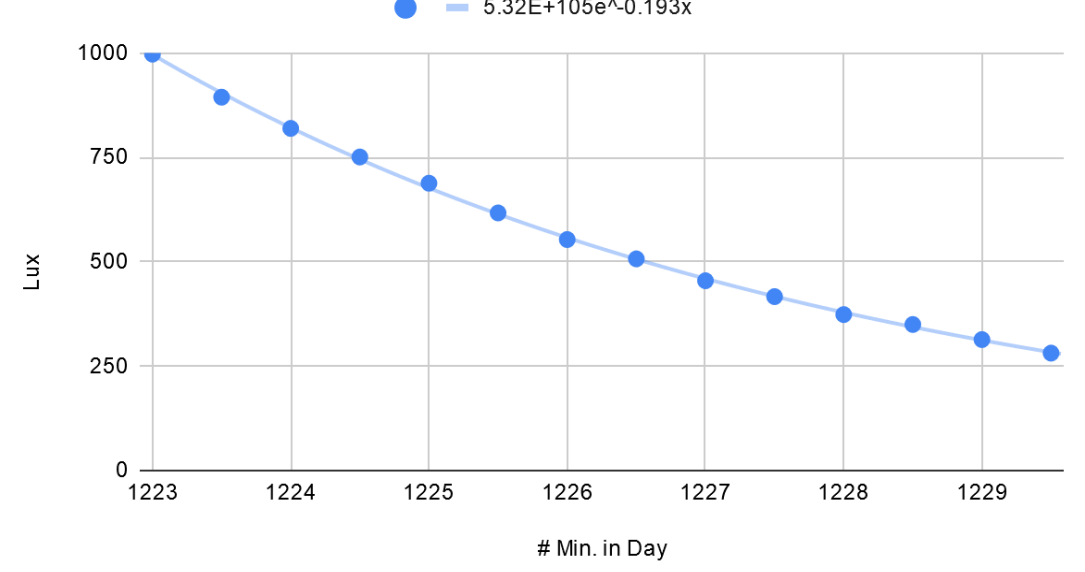

Fig. 10: Lux vs. Time (as converted to the number of the minute in the day) for sub-1000 lux samples

The exponential equation of the line of best fit in Figure 10 was used to extrapolate nighttime luminance values. Stationary deep-space objects (open clusters, globular clusters, and galaxies) were chosen as points of reference instead of moving celestial objects such as planets and asteroids for simplicity. Visible deep-space objects as logged on August 6 were recorded:

Table 3: Deep-space objects, their visual magnitude, starting time of luminance, and projected lux at that time Note: Data are from Ford $(2018)^{\mathrm{b}}$

\begin{tabular}{|l|r|l|l|l|}
\hline Object & \multicolumn{1}{|l|}{$\begin{array}{l}\text { Visual } \\
\text { Magnitude }\end{array}$} & $\begin{array}{l}\text { Time of } \\
\text { Visibility }\end{array}$ & $\begin{array}{l}\text { \# Min. in } \\
\text { Day }\end{array}$ & \multicolumn{2}{l|}{ Projected Lux } \\
\hline Triangulum Galaxy & $5.7^{\mathrm{b}}$ & $21: 05^{\mathrm{b}}$ & 1265 & 0.496 \\
\hline IC4665 (Open Cluster) & $4.2^{\mathrm{b}}$ & $21: 21^{\mathrm{b}}$ & 1281 & 0.0226 \\
\hline NGC6530 (Open Cluster) & $4.6^{\mathrm{b}}$ & $21: 21^{\mathrm{b}}$ & 1281 & 0.0226 \\
\hline
\end{tabular}




\begin{tabular}{|c|c|c|c|c|}
\hline NGC6633 (Open Cluster) & $4.6^{b}$ & $21: 21^{\mathrm{b}}$ & 1281 & 0.0226 \\
\hline Graff's Cluster (Open Cluster) & $4.6^{b}$ & $21: 21^{b}$ & 1281 & 0.0226 \\
\hline $\begin{array}{l}\text { The Sagittarius Cluster (Globular } \\
\text { Cluster) }\end{array}$ & $5.2^{b}$ & $21: 21^{b}$ & 1281 & 0.0226 \\
\hline M5 (Globular Cluster) & $5.7^{\mathrm{b}}$ & $21: 21^{\mathrm{b}}$ & 1281 & 0.0226 \\
\hline Great Globular Cluster in Hercules & $5.8^{\mathrm{b}}$ & $21: 21^{b}$ & 1281 & 0.0226 \\
\hline M12 (Globular Cluster) & $6.1^{\mathrm{b}}$ & $21: 21^{b}$ & 1281 & 0.0226 \\
\hline M3 (Globular Cluster) & $6.3^{b}$ & $21: 21^{\mathrm{b}}$ & 1281 & 0.0226 \\
\hline M15 (Globular Cluster) & $6.3^{b}$ & $21: 21^{b}$ & 1281 & 0.0226 \\
\hline M92 (Globular Cluster) & $6.5^{\mathrm{b}}$ & $21: 21^{b}$ & 1281 & 0.0226 \\
\hline M10 (Globular Cluster) & $6.6^{\mathrm{b}}$ & $21: 21^{b}$ & 1281 & 0.0226 \\
\hline Bode's Nebula (Galaxy) & $6.9^{b}$ & $21: 21^{b}$ & 1281 & 0.0226 \\
\hline Pinwheel Galaxy & $7.1^{\mathrm{b}}$ & $21: 21^{b}$ & 1281 & 0.0226 \\
\hline M94 (Galaxy) & $8.1^{\mathrm{b}}$ & $21: 21^{b}$ & 1281 & 0.0226 \\
\hline Whirlpool Galaxy & $8.4^{b}$ & $21: 21^{b}$ & 1281 & 0.0226 \\
\hline C7 (Galaxy) & $8.9^{b}$ & $21: 21^{b}$ & 1281 & 0.0226 \\
\hline Andromeda Galaxy & $3.4^{b}$ & $21: 25^{b}$ & 1285 & 0.0104 \\
\hline M2 (Globular Cluster) & $6.6^{\mathrm{b}}$ & $21: 26^{b}$ & 1286 & 0.00861 \\
\hline $\begin{array}{l}\text { Perseus Double Cluster - h Persei } \\
\text { (Open Cluster) }\end{array}$ & $4.3^{b}$ & $21: 33^{b}$ & 1293 & 0.00223 \\
\hline M110 (Galaxy) & $7.9^{b}$ & $21: 56^{b}$ & 1316 & 0.00002632 \\
\hline M32 (Galaxy) & $8.1^{\mathrm{b}}$ & $22: 02^{b}$ & 1322 & 0.00000827 \\
\hline $\begin{array}{l}\text { Perseus Double Cluster - Chi Persei } \\
\text { (Open Cluster) }\end{array}$ & $6.1^{\mathrm{b}}$ & $22: 15^{\mathrm{b}}$ & 1335 & 0.000000673 \\
\hline The Pleiades (Open Cluster) & $1.3^{b}$ & $23: 54^{b}$ & 1434 & 0.00000000000000339 \\
\hline
\end{tabular}

Visual magnitude of a celestial object is its brightness, with the object becoming fainter as its magnitude rises (Ford, 2019). No direct relationship was found between the magnitude of the visible objects and the projected lux as they became visible in the sky, nor was there a relationship between the type of object and their visibility. The average projected lux at the starting time of visibility was found to be roughly 0.036 lux. Given that the projected luminance in totality is $0.12 \mathrm{lux}$, this means that the average deep-space object will not be visible with the naked eye during the total eclipse, even in the path of totality, since the sky will not be dark enough. However, a rare exception may be made; as shown in the above chart, the Triangulum Galaxy became visible at a projected 0.5 lux, which would be visible in totality conditions.

Conclusion. Due to the complications posed by the COVID-19 pandemic, data collection was limited. The inability to launch a payload restricted the ability to test the experimental setup 
in flight conditions; more extensive testing can be done to ensure that it is viable for flight, such as ensuring temperature and weather resistance. Time and experiment formatting restrictions prevented extensive testing of the CHDK, so it would be of use to elaborate on the code created. This would include utilizing more of the CHDK library for finer control. As well, the remote triggering system for the camera could be expanded upon for weather resistance. Ideally the camera would be remotely triggered during flight to prevent hardware failure. Another important addition to this particular payload would be a stabilization system to ensure photo clarity; many of the space features imaged during such phenomena as the total solar eclipse are captured with long camera exposures which, with instability, will render blurry photos. Regarding luminance, it would be valuable to collect data across a whole, clear day (as close to flight conditions as possible) in order to verify the accuracy of the extrapolated mathematical relationships found.

\section{Ionosphere}

The Ionosphere sub-team's goals were to observe how the magnetic fields in the ionosphere change with the amount of sunlight. This would give us insight into how ionospheric magnetic fields will behave during a solar eclipse.

Introduction. The ionosphere is the region in the atmosphere that contains charged particles of ions and electrons. It is created when radiation from the sun knocks electrons free from the atoms which results in ions and free electrons. One important application is that radio signals can be sent to the ionosphere, and these will bounce back when the frequency of the radio pulse matches the resonant frequency of the local electrons. The ionosphere overlaps with the magnetosphere, which is the region where the charged particles are greatly affected by the Earth's and the Sun's magnetic fields. Because the ionosphere is so reliant on the sun's radiation, it is expected that data collected on magnetic fields at a high altitude will vary depending on how much solar radiation reaches the earth. A solar eclipse provides a unique opportunity to observe this.

Methods. To observe the magnetic field, the team experimented with a magnetometer, the Adafruit LSM303. This sensor can be used to find magnetic north when there are no other magnetic influences around. Some things that can affect the data are nearby magnetic material or high-powered circuits. The magnetometer was programmed to record magnetic strength in micro teslas from the $\mathrm{X}, \mathrm{Y}$, and $\mathrm{Z}$ vectors as well as output the compass heading, which shows the direction the sensor is facing. Several experiments were completed with the magnetometer. Data was collected by rotating it in different directions, tilting it, shaking it, and exposing it to magnets and high-powered circuits. These experiments were necessary to analyze how the sensor readings will be affected by factors such as motion and tilt during the payload's ascent. They were also necessary to determine where the sensor should be placed within the payload.

In order to observe solar radiation, an experimental set-up using an IR sensor, photoresistor, and a solar panel was assembled. Data from the IR sensor and the photoresistor was used to cross check the data from the solar panel and confirm that the measurements make sense.

A solar panel will be used to collect data on solar radiation during the flight, and the data that will give us a more accurate look on the amount of solar radiation will be power (voltage $\mathrm{x}$ 
current). Data was collected during late morning/high noon (10:30-12:00) to see what it looks like when the sun is fully out and late afternoon/sunset (19:00-21:30) to see what the data looks like when there is little sun, as there would be during a solar eclipse.

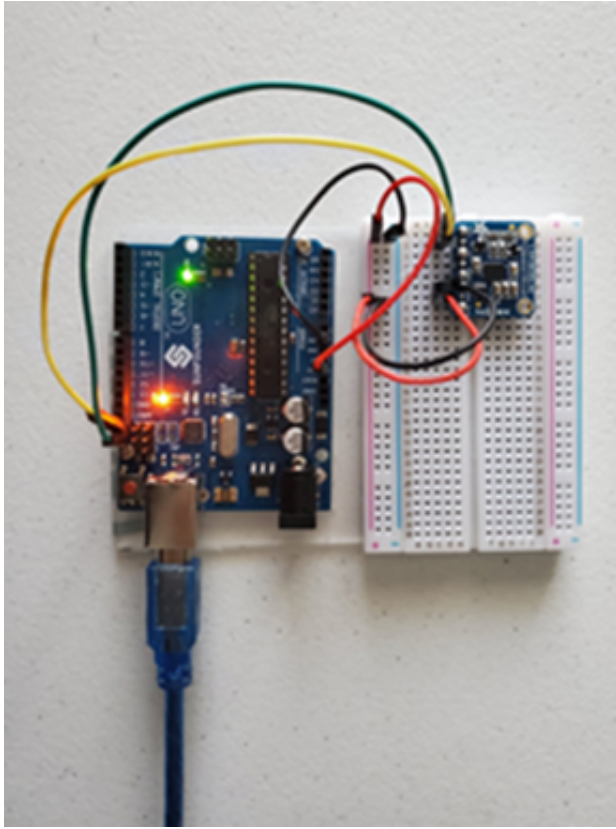

Fig. 11: LSM303 Magnetometer connected to the Arduino

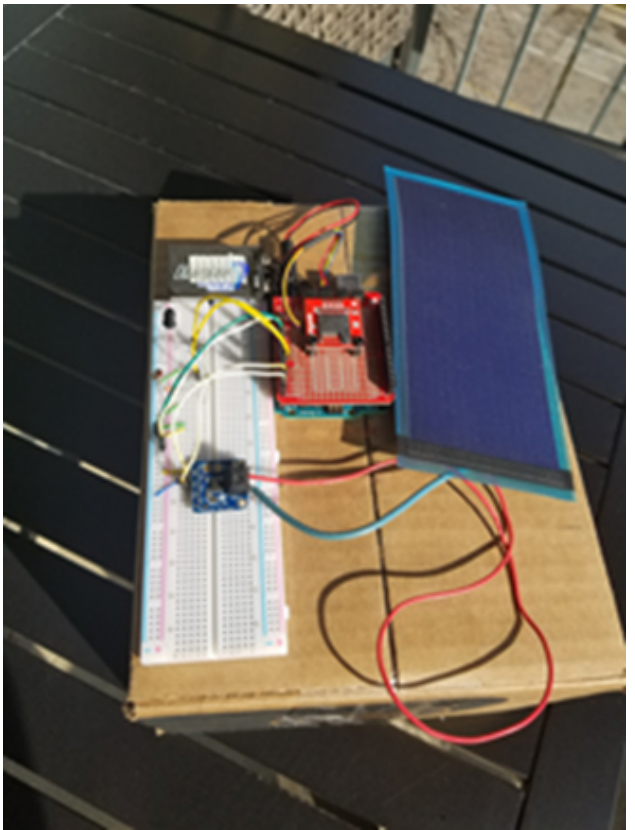

Fig. 12: Experimental set-up with IR sensor, photoresistor, and solar panel connected to the Arduino

Results. The magnetometer observed changes in values from tilt, motion, and nearby magnetic influence. There are sudden leaps in the values when there is a nearby magnetic influence from a refrigerator magnet. This is theoretically similar to the battery pack that would have been in the payload. Each row in Tables 4 and 5 represent a time step. The magnet was brought one centimeter away from the side of the sensor, resulting in the large value in the first row of Table 4. The magnet was then moved to the topside of the sensor. Then the magnet was pulled away resulting in the values on row four and five of Table 4 which are close in value to each other.

Table 4: Magnetometer Readings

\begin{tabular}{|r|r|r|r|}
\hline $\begin{array}{c}\text { Magnetic X } \\
(\mu \mathrm{T})\end{array}$ & $\begin{array}{c}\text { Magnetic } \mathrm{Y} \\
(\boldsymbol{\mu} \mathrm{T})\end{array}$ & $\begin{array}{c}\text { Magnetic } \mathrm{Z} \\
(\boldsymbol{\mu} \mathrm{T})\end{array}$ & $\begin{array}{c}\text { Compass } \\
\text { Heading }\end{array}$ \\
\hline 501.74 & 430 & 51.22 & 40.6 \\
\hline 431.74 & 241.74 & 55.12 & 29.25 \\
\hline-108.7 & -79.13 & 71.22 & 216.05 \\
\hline 11.74 & 8.26 & 28.29 & 35.13 \\
\hline 10.43 & 10 & 28.29 & 43.78 \\
\hline
\end{tabular}

Table 5: Tilted Magnetometer Readings 


\begin{tabular}{|r|r|r|r|}
\hline $\begin{array}{r}\text { Magnetic X } \\
(\boldsymbol{\mu} \mathrm{T})\end{array}$ & $\begin{array}{r}\text { Magnetic } \boldsymbol{Y}(\boldsymbol{\mu} \mathrm{T}) \\
\hline 29.64\end{array}$ & $\begin{array}{c}\text { Magnetic } \mathbf{Z} \\
(\boldsymbol{\mu} \mathrm{T})\end{array}$ & $\begin{array}{r}\text { Compass } \\
\text { Heading }\end{array}$ \\
\hline 43.91 & 26 & 26.94 & 41.26 \\
\hline 56.64 & 15.82 & 30.61 & 30.89 \\
\hline 61.64 & 15 & 32.96 & 15.6 \\
\hline 71.27 & 12.45 & 43.88 & 13.68 \\
\hline 79.27 & 8.45 & 51.53 & 6.91 \\
\hline 85.18 & 9.27 & 63.78 & 6.21 \\
\hline 92 & 6.09 & 80.41 & 3.79 \\
\hline 92.55 & 3.82 & 97.65 & 2.36 \\
\hline 86.55 & 2.45 & 122.86 & 1.62 \\
\hline 68.82 & 3.55 & 145.71 & 2.95 \\
\hline 45.09 & 8.91 & 158.88 & 11.18 \\
\hline 20.91 & 17.18 & 160.1 & 39.41 \\
\hline-1 & 17.64 & 156.53 & 93.25 \\
\hline-12.73 & 18.18 & 150.61 & 124.99 \\
\hline-14.73 & 18.27 & 149.08 & 128.87 \\
\hline-17.27 & 19.55 & 147.24 & 131.47 \\
\hline-33.64 & 8.91 & 134.8 & 165.17 \\
\hline
\end{tabular}

It is expected that the data during the ascent of the payload will not deviate much from data collected at peak altitude because that is when the sun will be covered and the ionosphere layers stripped.

The data from the solar radiation experimental set-up was just as expected. For each sensor, the values decreased as the sky's brightness decreased, and increased as the sky's brightness increased.

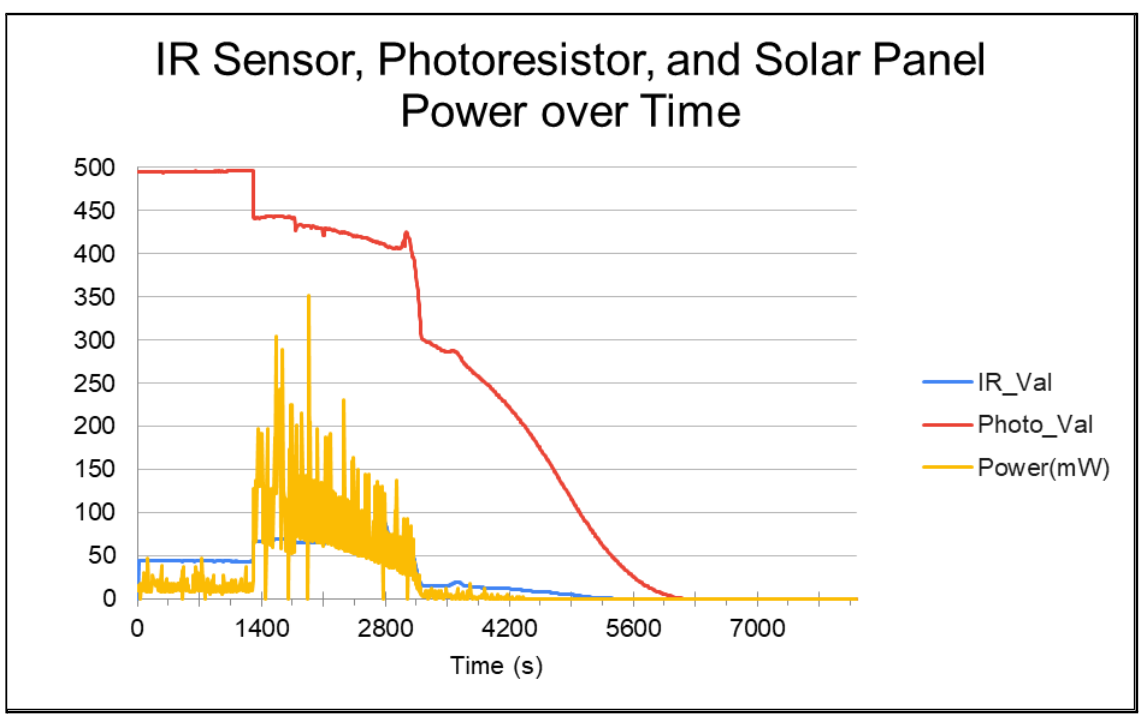

Figure 13: Data collected from 19:00 to 21:30. Values decreased as the sun sank below the horizon. 


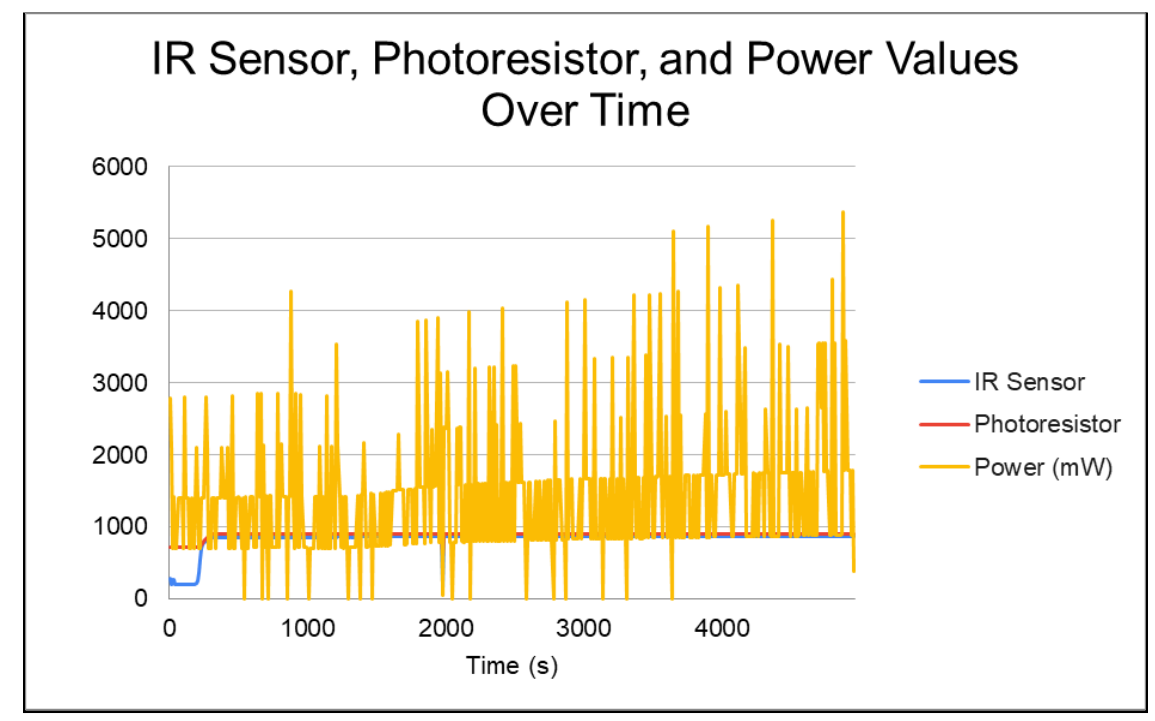

Figure 14: Data collected from 10:30 to 12:00. Power slightly increased as the time approached high noon.

Conclusion. Before the 2024 Solar Eclipse, more work needs to be accomplished. First, the accelerometer built in the LSM303 needs to be integrated in the code to compensate for tilt. Accelerometer data also allows us to cross-check sudden jumps in the data from the magnetometer because it measures gravity in the $\mathrm{X}, \mathrm{Y}$, and $\mathrm{Z}$ vectors. The magnetometer should be placed as far away from the battery pack as possible to limit its influence on the sensor's data collection. Second, there needs to be experimentation conducted that will test how extreme cold will affect the performance of the solar panel because the solar panel will be placed on the exterior of the payload. This is important because the solar panel's operating temperature is at minimum $-40^{\circ} \mathrm{C}$ but the outside atmospheric temperature can reach up to $-70^{\circ} \mathrm{C}$. One suggestion for the experiment is to use dry ice. These suggestions, along with more test runs, will allow the final data collected to be more reliable for future analysis that will lead to better insight into the ionosphere.

\section{Acknowledgements}

The authors would like to thank Dr. William Farrow for acting as advising professor during this research term, as well as team lead Daniel Schuler, and team members Emily Harper, Julia Kalish, Jesse Lambrecht, and Bella Ness for their ample contributions to this project.

\section{References}

Ford, D. (2018, August 1). Objects in your sky: Deep sky objects. In-The-Sky.org. Retrieved August 6, 2020, from https://in-the-sky.org/data/deepsky.php

Ford, D. (2019, July 26). Magnitude. In-The-Sky.org. https://in-the-sky.org/article.php?term=magnitude Hamblen, J. (2015, September 15). Using a photocell or Phototransistor to determine lighting levels. Free open source IoT OS and development tools from Arm | Mbed. Retrieved August 25, 2020, from https://os.mbed.com/users/4180_1/notebook/using-a-photocell-to-determine-light-levels/ Pettengill, R. (2017, September 8). How much reduction in sunlight occurs during a total solar eclipse? Quora. Retrieved August 25, 2020, from https://www.quora.com/How-much-reduction-in-sunlight-occurs-during-a-total-solar-eclipse University of Tennessee Institute of Agriculture. (n.d.). Sunlight. https://ag.tennessee.edu/solar/Pages/What\%20Is\%20Solar\%20Energy/Sunlight.aspx 